\title{
Safe Removal of Combat Body Armour Lightweight Following Battlefield Wounding - A Timely Reminder
}

Col J M Ryan

MCh, FRCS, late RAMC

Maj R Bailie

FRCAnaes, RAMC

Maj G Diack

$M B, B S, R A M C$

Capt J Kierle

RGN, QARANC

Cpl T Williams

OTT 1, RAMC

MST Bravo, Operation Grapple 2, PWO Group, Vitez School, BFPO 547

SUMMARY: Medical Support Troop Bravo (MST Bravo) provides third line medical support for the United Kọlg dom Battle Group currently deployed on operation Grapple 2 in Bosnia Herzegovina. Standing operating proc dures for the initial assessment and resuscitation of all casualties are based on the protocols taught on Advance? Trauma Life Support (ATLS) and Battlefield Advanced Trauma Life Support (BATLS) courses.

These protocols dictate a period of initial assessment and resuscitation with the casualty immobilised in supine position. Recent experience in casualty management at MST Bravo has identified inadvertent spinal mô ment taking place during removal of Combat Body Armour Lightweight (CBA) which might have detrime@t effects for casualties with occult spinal injury. This has led to an appraisal of CBA removal and the identifica 900 of a drill for rapid, safe and reliable means of undressing without jeopardy to the integrity of the spine. method is described.

\section{Introduction}

Since 1985 the DMS has established standardised criteria for early management of battle casualties. This followed the introduction of the BATLS package by the then Professor of Military Surgery. Derived from the North American ATLS programme, BATLS protocols dictate not only the pace and intensity of early management, but also the sequence of activities to be undertaken and the position in which casualties are to be maintained (Table 1). Casualties must be kept in the supine position during the conduct of the primary survey and resuscitation phases. Full immobilisation of the whole spine is stressed with particular emphasis on maintaining the integrity of the cervical spine (Table 2). Although a counsel of perfection for the doctor or combat medical technician working alone in the forward areas (vertical management), it should be aimed for in the MST or Field Hospital setting where a team based or horizontal approach to early management is usually possible.

\section{Materials \& Methods}

MST Bravo receives a steady trickle of casualties from own forces and the local population. Battle Group casualties invariably arrive clad in CBA and wearing helmets. A
Table 1.

Steps in Initial Assessment \& Early Management

1. Rapid primary survey

2. Resuscitation

3. Secondary survey

4. Initiation of definitive care

* Steps 1 \& 2 conducted concurrently

Table 2.

The Primary Survey
A - Airway maintenance and cervical spine control
$\mathrm{B}$ - Breathing and ventilation
C - Circulation with haemorrhage control
D - Disability - neurological status
E - Exposure - environmental control - evacuation

recent incident involving a Warrior AFV rolling over on its turret resulted in the admission of three casualties 8 the resuscitation area of the MST. Initial assessment ard 
early management was carried out by teams working horizontally to a predetermined plan. Team members have individual responsibility for particular components of the Primary Survey (Table 2). The task of exposing casualties is, by convention, carried out by an OTT using stretcher bearer scissors, and is performed concurrent with other activities. No problem is encountered when removing conventional combat clothing but difficulties may arise in removing closely fitting and well secured CBA (Fig 1). We have found this material resistant to cutting with scissors leading to efforts at normal removal which results in unacceptable casualty movement (casualty sat up or rolled).

\section{Combat Body Armour Lightweight (CBA)}

United Kingdom personnel deployed on operational tours are required to wear head and torso protective equipment. The aim is to defeat missiles of low available energy (low velocity bullets and modern preformed fragments). No protection is afforded against penetration by missiles with high available energy (high velocity bullets

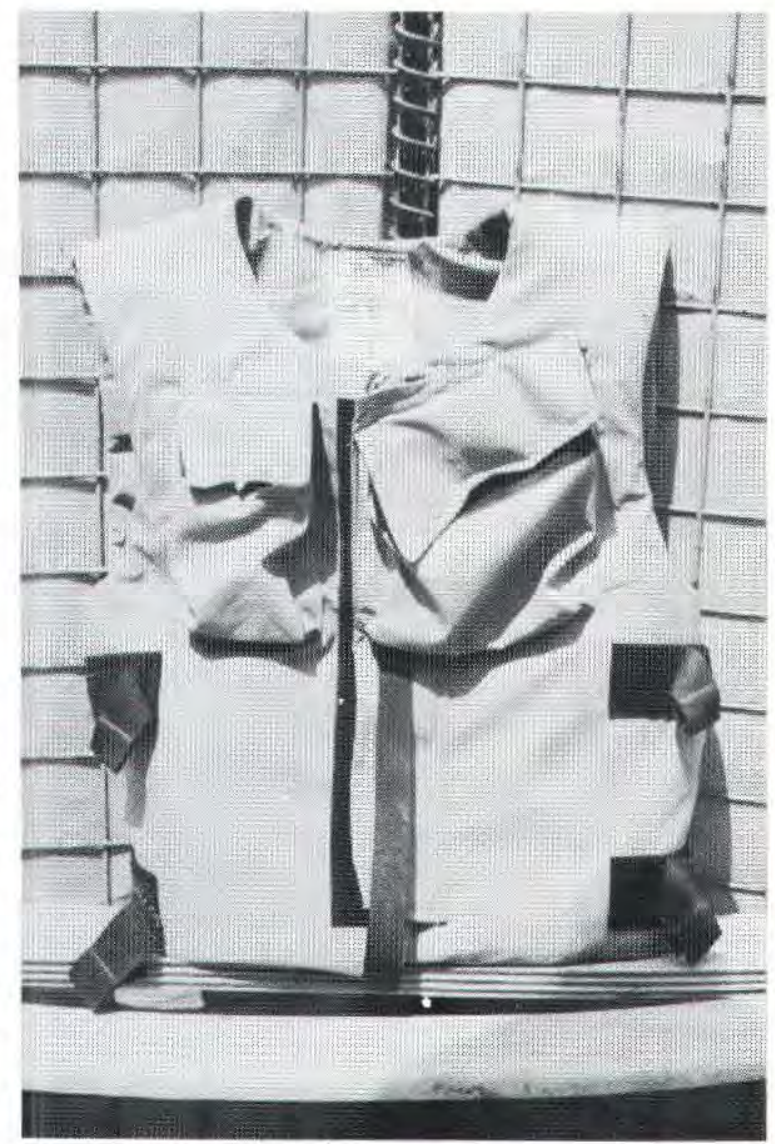

Fig 1. Combat Body Armour Lightweight (CBA). and fragments with high available energy). Torso protection is afforded by wearing a sleeveless vest consisting of layered kevlar enclosed within a waterproof envelope; the whole is covered in an appropriate operational fabric (Fig $1)$. The vest has a front panel with a centrally placed velcro fastener which must be opened to allow normal removal. A rear panel protects the back and is connected to the front panel by two fabric tabs and two adjustable side straps with velcro fasteners. The front and rear panels are in continuity over the shoulders.

\section{Removal of CBA - A Safe Method}

Following their recent experience in removing CBA from injured servicemen, MST Bravo personnel carried out a field study aimed at removing equipment without casualty disturbance. Stretcher bearer scissors were found to be effective in the hands of a robust male OTT, providing the scissors were razor sharp - in other circumstances they failed to cut cleanly resulting in tearing and shearing with considerable casualty movement. Plaster shears and saws also resulted in tearing and shearing and were

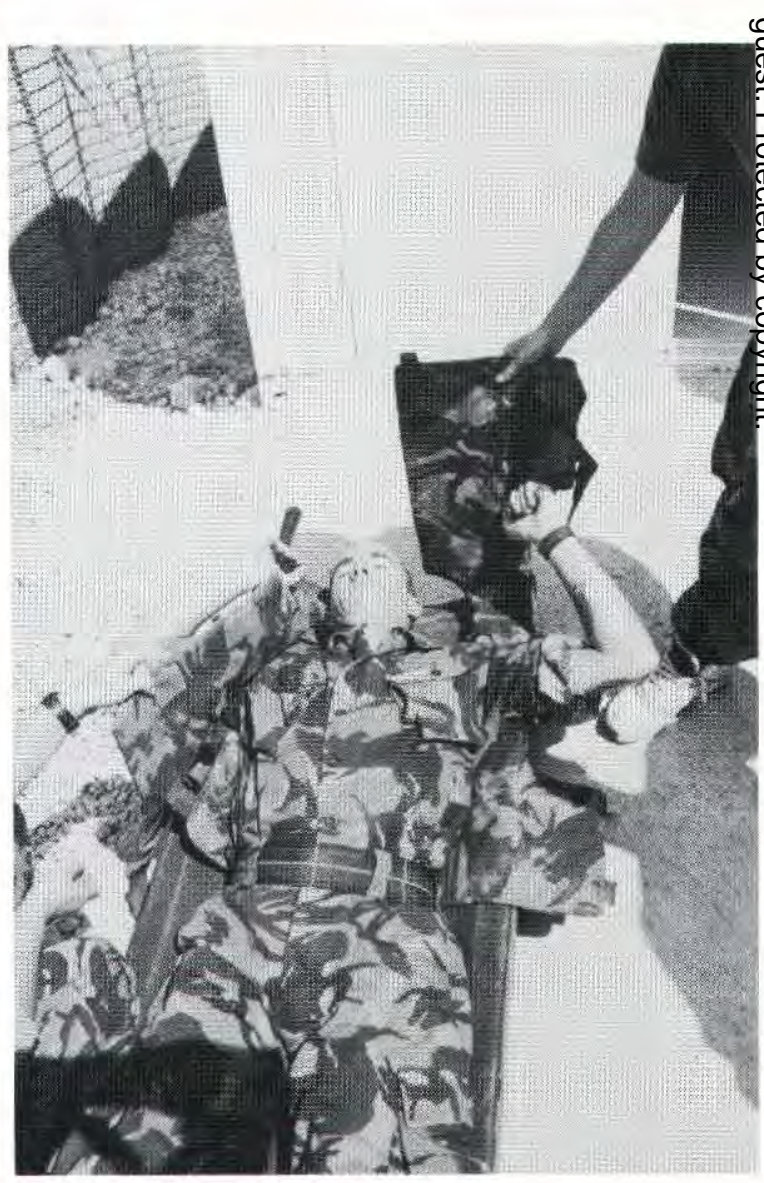

Fig 2. The steps in removing CBA.

\section{r}

(1)

윽

ఫิ

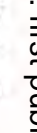 \\ ज़}


deemed unsatisfactory. Gigli saws were used and found to be effective but required considerable force resulting in casualties being lifted from the stretcher - the method was abandoned. Sequential opening of velcro flaps and normal removal required casualties to be standing or sitting and is unacceptable. The safest means and the one recommended involves;

a. opening the anterior, centrally placed velcro fastener.

b. turning the right and left halves of the anterior panel out to the sides exposing the paired, sewn fabric tabs.

c. cutting the paired tabs with scissors exposing the laterally placed, adjustable velcro straps.

d. opening and detaching the lateral straps without casualty movement.

e. reflection upwards of the now free anterior panels exposing the anterior torso, permitting the primary survey to proceed unimpeded.

Figure 2 illustrates the steps. The anterior velcro fastener has been opened. The right half of the anterior panel has been turned out to the side exposing the sewn fabric tabs.

On the left, the tabs have been cut, the adjustable lateral straps have been opened and detached allowing the patel to be freely reflected upwards.

The casualty is now lying supine on the posterior pisel which should remain in place until the casualty is $\mathbb{R}$ rmally log rolled at a convenient moment during the $\Omega_{c}$ ondary survey phase of initial management.

\section{Conclusions}

Under field conditions, spinal injury cannot be exclum until a casualty reaches a definitive facility, normallo at 4th line. Yet ATLS and BATLS protocols demand ce피plete immobilisation of the entire casualty at all tios until a spine injury is excluded, especially when a c: $\mathbb{\otimes}-$ alty is to be transferred or evacuated. This is a diffigitt task in the field setting and may be impossible in forwe क्षु areas. However, occult spinal injury must be considered and all reasonable means taken to minimise casuabty movement. We describe a rapid, safe and easily app $\mathbb{R}$ d means for removing CBA which achieves the aim of nơ imising movement and allows the primary survey and resuscitation phases to proceed according to protocol. believe it to be particularly appropriate when dealing with unconscious casualties or for those with overt spimal involvement where the risk of secondary neurol $\frac{10}{\sigma} \delta$ al injury is considerable. 